\title{
Self-Extubation in the Surgical Intensive Care Unit and Restraint Policy Change: A Retrospective Study
}

\author{
John T. Denny ${ }^{*}$, , Enrique Pantin ${ }^{1}$, Julia Denny ${ }^{2}$, William Grubb ${ }^{2}$, James Tse ${ }^{1}$, Darrick Chyu ${ }^{1}$, \\ Phat Trihn ${ }^{1}$ and Christine Hunter ${ }^{1}$
}

\author{
${ }^{I}$ Robert Wood Johnson Medical School/Rutgers University Dept. of Anesthesia, 125 Paterson Street, New Brunswick NJ \\ 08901, USA \\ ${ }^{2}$ Rutgers School of Nursing Graduate Program in Nurse Anesthesia, USA
}

\begin{abstract}
Background: Self-extubation is a serious complication of mechanical ventilation. The incidence of selfextubation in a university hospital may be altered by a recent policy change, which requires daily written and face-to-face patient re-evaluations regarding the need for restraints in the intensive care units. There are many concerns and suspicions that this might result in less use of restraints and thereby increase the risk of self-extubation.

Methods: The goal of this study was to describe the incidence of self-extubation (SE) over time and report any observed change with the change in restraint policy (RP).

Results: The rate of self-extubation during the control year was $1.62 \%$ which increased to $2.33 \%$ during the initial study period and decreased to $1.30 \%$ in the following year. The distant follow-up period eight years after the initial change in restraint policy showed a rate of $1.14 \%$. Apache scores did not differ significantly between the years compared.

Conclusion: A new restraint policy, which requires frequent reordering by physicians, has the potential to be associated with increased self-extubation, at least temporarily. Staff education may help reduce this risk. Further research will be useful in clarifying which interventions can most reduce this potentially life-threatening complication.
\end{abstract}

Keywords: Complications of endotracheal intubation, ICU complications, self-extubation, unintended extubation, unplanned extubation complications of mechanical ventilation, ventilator weaning.

\section{BACKGROUND}

Self-extubation, the process whereby an intubated patient removes his own endotracheal tube (either deliberately or accidentally), is one of the most common clinical problems associated with mechanical ventilation, along with ventilator associated pneumonia and laryngeal damage. Under normal conditions, the process of weaning a patient from mechanical ventilation constitutes a significant portion of the time a patient is in the ICU. Before weaning, ICU nurses, physicians and respiratory therapists must carefully consider the state of the patient's airway, the ability of the patient to clear secretions and protect the airway, the patient's mental status and the absence of respiratory compromise [1]. Selfextubation can compromise the patient's health and complicates the safe and gradual process of mechanical ventilation weaning. Although some literature exists that describes the problems of self-extubation in detail, the literature lacks some data on risk factors associated with the likelihood that a patient will self-extubate [2].

*Address correspondence to this author at the Robert Wood Johnson Medical School/Rutgers University Dept. of Anesthesia, 125 Paterson Street, New Brunswick NJ 08901, USA; Tel: 732235 6268; Fax: 732235 6131;

E-mail: dennyjt@rwjms.rutgers.edu
The control period reviewed is year one, during which the hospital in this study followed the old restraint policy. During this year physicians ordered restraints verbally. In year two, the hospital modified its previous policy to require daily patient re-evaluation and daily re-ordering of patient restraints. This new policy led to a concern that there would be an increase in self-extubation.

The State Health Department changed its regulations regarding the ordering of patient restraints. As a result, the hospital's policy also changed. In year two, the hospital required doctors to write restraint orders instead of verbally ordering restraints. In addition, daily face-to-face patient reevaluations were required in order to determine the ongoing need for restraints. This led to concern that the incidence of self-extubation might increase.

\section{OBJECTIVE}

The goal of this study is to describe the incidence of selfextubation (SE) over time and report any observed change with the change in restraint policy (RP).

\section{METHODS}

We conducted an IRB-approved retrospective record review utilizing the records of patients admitted to the Surgical and Trauma Intensive Care Unit of a Level One 
Trauma Center University Hospital during years one, two and three. This particular Surgical and Trauma ICU consisted of 17 beds and included patients from Trauma, General Surgery, Oncology Surgery, Orthopedics, Urology, and Neurosurgery. Physician coverage was provided 24 hours a day by surgical and anesthesia residents. The average ICU nurse to patient ratio was 1: 2 patients.

In this institution, intubated patients were routinely sedated with various combinations of intravenous midazolam, fentanyl, and/or propofol. The combination of drugs used for sedation of each patient remained constant throughout the periods studied.

Data retrieved from year one medical records served as our baseline data. Medical records from year two comprised the initial study period. We also reviewed records from year three to see if we could visualize a trend. We computed the rates of self-extubation against days on a ventilator to determine whether staff familiarity with the restraint policy affected the incidence of self-extubation. A follow-up review was also conducted eight years later.

The Director of the Department of Statistics and a Professor from the same department analyzed the data. We utilized the statistical test for relative risk using 95\% confidence intervals, with reference to Rothman [3].

We excluded records that described accidental extubations (AE) resulting from nurse-turning or patient transportation. The number of patients who extubated themselves was divided by the number of patient days on the ventilator (ventilator days), giving a percentage we call 'Rate of Self-Extubation'. The denominator thus, standardized the amount of ventilator usage.

\section{RESULTS}

Table 1 shows the self-extubation rates for the years one, two, three, and eight years later.

The rate of self-extubation during the control year (year one) was $1.62 \%$ increasing to $2.33 \%$ during the initial study period (year two) and decreasing to $1.30 \%$ in the following year (year three). The distant follow-up period eight years after the initial change in restraint policy showed a rate of $1.14 \%$. Apache scores did not differ significantly between the four study years compared. Rate of extubation is calculated as (self-extubations per year) divided by the (number of days on a ventilator in a year).

\section{DISCUSSION}

Literature regarding self-extubations rates in intensive care units reports widely varying rates. A study by Schwartz et al., which examined the rate of self-extubation in tertiary care intensive care units found self-extubation rates of $7.3 \%$. Accidental extubations accounted for $22.1 \%$ of the incidents and deliberate self-extubations accounted for $77.9 \%$ of the incidents [4]. In one study, the rate of unplanned extubations in the surgical ICU was 3\% per year. Deliberate selfextubations accounted for $87 \%$ of these incidents [4]. Another study by Chevron et al. in a University intensive care unit reported a self-extubation rate of $14 \%$ [5]. A third study by Vassal et al. reported a self-extubation rate of $12 \%$ in studies targeting infant and child tertiary care populations [5], Rivera and co-workers reported a self-extubation rate of $3.2 \%$ and Little et al. reported a rate of 1.15 accidental extubations (AE) per 100 intubated days in their PICU and reported a rate of $0.72 \mathrm{AE}$ per 100 intubated days in their NICU $[6,7]$.

There are several reasons why patients in the ICU may self-extubate. Studies and experience show that not using physical restraints designed to prevent upper extremity movement is a major cause of self-extubation. Another contributor is discomfort from the presence of invasive lines or endotracheal tubes. Another factor is a shortage of staff. Beckman and Chang found that self-extubations were more likely to occur on days when staffing was lower [8]. Certain ICU patient groups have been shown to experience selfextubation at a higher rate. Achauer and colleagues found a high extubation rate of $27 \%$ in burn patients compared with a rate of only $6 \%$ in the general surgery ICU population [9]. Burn victims who undergo painful or invasive procedures have an especially high rate (66\%) of extubation [9].

Research has also identified what does not seem to cause an increase in self-extubation. A study by Atkins et al. showed that the presence of restlessness, agitation or the presence of an adverse event in the hospital does not increase the incidence of self-extubation [10]. However, Lucas da Silva found that an increased level of consciousness was a risk factor for unplanned extubation [11]. A study by Christie et al. reported that the time of day had no impact on the rate of self-extubation. In their study, $85 \%$ of the unplanned extubations were deliberate self-extubations and $15 \%$ were accidental [12]. Curry's study also showed no difference in the rate of unplanned extubations between the day and night shift [13]. The study by Chevron et al. found that nursing workload did not seem to play any role in

Table 1. Self-Extubation Rates: Years one, two, three, and eight years later.

\begin{tabular}{|c|c|c|c|c|}
\hline Year & Self Extubations Per Year & Number of Days on Ventilator Per Year & Rate of Self-Extubation (\%) & p-Value $\boldsymbol{v s}$ Year-One \\
\hline \hline Year one (Control) & 28 & 1725 & $1.62 \%$ & $2.33 \%$ \\
\hline Year two (RP change) & 71 & 3047 & $1.30 \%$ & $<0.05 *$ \\
\hline Year three & 48 & 3690 & $1.14 \%$ & NS \\
\hline Eight years later & 43 & 3797 & NS \\
\hline
\end{tabular}

Remarks:

$\mathrm{RP}=$ Restraint Policy

NS $=$ Not Significant

$\mathrm{N} / \mathrm{A}=$ Not Applicable.

$* \mathrm{p}<0.05$ is considered significant. 
whether patients would self-extubate or not [14]. According to Frank et al., smaller unit size and lower acuity were also not associated with increased accidental extubations [15].

Any type of extubation for any reason can result in potential harm to the patient. Self-extubation in intensive care units may be accompanied by complications that include difficult re-intubation. Even initial intubation of critically ill adults is associated with major complications. Re-intubation was accompanied by hemodynamic or airway complications in $72 \%$ of the cases in one study. Another study by Mort points out that the actual rate of complications is likely higher than the above self-reported rate of $72 \%$ [16, 17]. Hospitals that are Level One trauma centers, similar to the setting of this study, are not immune from unwanted complications of mechanical ventilation [18]. Not surprisingly, patients that do not require re-intubation after self-extubation have significantly lower inspired oxygen concentrations and ventilator delivered minute volume than those requiring re-intubation [17].

A proactive approach to patient extubation is important in order to improve quality of care [19]. It has been proposed that decreasing the length of the weaning process, and extubating as soon as medically possible will reduce unplanned self-extubations in the ICU.

Self-extubation has been linked to a variety of factors. One example is a lack of sufficient staffing. Beckman et al. and Chang found that self-extubations are more likely to occur on days when staffing is lower $[8,20]$. When fewer nurses are present, it is less likely that a nurse is able to, in a timely manner, take care of a patient that has become free of their restraints. The patient's arms are thus, free to dislodge the endotracheal tube either intentionally or accidentally. Furthermore, the risk of self-extubation increases if nurses do not place any restraints on a patient. Tominaga et al. reported that when their hospital policy restricted the use of hand restraints, self-extubation rates significantly increased [21]. Brandstetter et al., reported that restraints were more effective than sedation in preventing repeated accidental extubation [22].

As illustrated in our results, the baseline rate of selfextubation in year one was $1.62 \%$. The rate rose to $2.33 \%$ in year two immediately after a restraint policy change. The rate decreased to $1.30 \%$ in the year three. The rate further decreased to $1.14 \%$ in distant follow-up data eight years post restraint policy change. The data suggest that an initial increase in self-extubation rates occurred following the restraint policy (RP) change. This initial increase was, interestingly, followed by a decrease in self-extubation rate to below baseline levels once the staff fully adopted the new restraint policy.

Healthcare quality control surveys increasingly consider unplanned extubations as indicators of ICU quality. The unplanned extubation rate varies from $3 \%$ to $14 \%$ in the ICU with burn ICU patients rating the highest (27\%) [9]. Reintubation rates typically vary widely, from $31 \%$ to $74 \%$, depending on need and the condition of the patient. Preventive strategies are essential to decrease these selfextubation rates. First, each hospital should analyze its unique setting in order to plan future action. This analysis should take into consideration patient safety and quality of patient care. Hospitals need to put preventative measures in place that will mesh with their analysis. Preventative measures should include providing staff education, increasing staff during nursing interventions, researching for risk indicators of self-extubation, using effective sedation protocols, and finally utilizing alternative protocols that can take the place of physical and pharmacological restraints. Furthermore, the relationship between unplanned extubations and nursing workload should and can be used as indicator for the optimization of staff resources in intensive care unit [23].

The perceived value that hospitals have of improved patient comfort and autonomy has driven recent attempts to minimize the use of restraints. When the restraint policy changed, staff were likely unfamiliar with the new protocols and requirements for ordering restraints. Thus, it is not surprising that the rate of self-extubation increased immediately after hospitals changed the restraint policy. The result was that more patients likely did not have their restraint orders renewed in a timely manner. More importantly, we were encouraged by the decrease in the rate of self-extubation to below baseline levels once the staff had time to become familiar with the new restraint policy.

Other hospital policies besides the restraint policy affect self-extubation rates as well. Weaning protocols are associated with decreased incidence of unplanned extubation [24]. A study by Jarachovic et al. showed that weaning protocols were a significant predictor of unplanned extubation; that patients who had weaning protocols ordered and followed were least likely to experience unplanned extubation. Thus, the use of standardized protocols is deemed useful for health care providers [25].

Another factor important to the prevention of selfextubation is the existence of a quality improvement program and the implementation of standard protocols in the hospital. A study by Rachman et al. determined the rate of unplanned extubations and contributing factors and determined whether a targeted intervention program would be successful in decreasing the rate of unplanned extubations in a 10-bed Pediatric Intensive Care Unit (PICU). They concluded that the rate of unplanned extubation in a PICU can be decreased with a quality improvement program that targets the institution's specific needs [26]. Their findings were similar to that of Sadowski et al. in 2004 [27]. Tindol et al. described that most hospitals had no standard operating procedure for managing unplanned extubations. Useful protocols should include the assessment of arterial blood gas, clinical evaluation of the patient's respiratory status, and assessment of the patient's mental and overall clinical status. A larger patient study population is required to determine the validity of such guidelines [28-32].

Other factors include poor collaboration and ET care. Poor professional collaboration can negatively affect the delivery of healthcare services to patients. Practice-based interventions can improve both professional practices and healthcare outcomes [33]. Although several studies have been conducted on various aspects of ET care, no clear standard of care on intubated patients has emerged. Future research findings should be utilized to ensure effective nursing interventions, minimize complications, and provide optimal nursing care [34]. 
There are important limitations to the study conducted in this paper. First, it is a retrospective study without a proper control group. It is not possible to state a specific cause and effect between the restraint policy change and the rate of self-extubation. We can only state that a tightening of the restraint policy is associated with a temporary increase in the rate of self-extubations. Unfortunately, it was not possible to quantitate the actual amount of restraint use from patient charts, information that would have been valuable both for this research and for future patient care. The hospital was unable to provide information on the depth of sedation, such as the Ramsey score, for the years in question. Second, we were thus, unable to gauge the adequacy or depth of sedation of intubated patients. Third, we were unable to find data on the frequency of re-intubation or information on morbidity related to self-extubation. In the future, researchers need to fill in these holes in order to clarify if additional interventions not considered in this paper, can further reduce the rate of self-extubation [35]. Another limitation we encountered was the fact that data on self-extubation in the literature is disparate and not reported in a uniform manner. For example, some centers report number of selfextubations/number of intubated patients; some centers report number of self-extubations per ventilator days; some report the number of self-extubations per days intubated; and yet others report the number of self-extubated patients intubated. Reporting in a standardized fashion might allow for a more meaningful comparison of different strategies and techniques in reducing self-extubation. Reporting the number of self-extubations/per ventilator days or number of days at risk is one useful description [36].

Medical terminology is also disparate, with some authors referring to accidental extubation, and others referring to self-extubation. This report looks only at self-extubations, and excludes accidental extubations.

The phenomenon of accidental, mechanical extubation (AE) is mechanistically unrelated to patient self-extubation. Accidental extubation can be due to factors unrelated to patient sedation, comfort, or restraint use (e.g. extubation while turning or transporting patients or performing diagnostic studies). Therefore, it would be useful to report the two phenomena separately and distinctly. In addition, accidental extubations could be excluded and reported separately as number of accidental extubations per number of ventilator days. Simple disconnections of the ventilator circuit from the tracheostomy could also be excluded, or reported separately since the airway is not technically lost as it is with self-extubation, or with removal of the tracheostomy itself. Standardization of reporting is the first step in being able to meaningfully compare rates between different centers, and to compare the results of different interventions to reduce self-extubation.

This paper finds that restraint policy that requires frequent reordering by physicians has the potential to be associated with increased self-extubation, at least temporarily. Staff education may help reduce this risk. Further research will be useful in clarifying which interventions can most reduce this potentially lifethreatening complication.

\section{SUMMARY OF KEY POINTS}

Unplanned self-extubation is a serious complication of mechanical ventilation. The incidence of self-extubation in a university hospital was before and after a policy change that required daily re-evaluations on the need for restraints in the intensive care units. This new restraint policy also requires frequent reordering of restraints by physicians and has the potential to be temporarily associated with increased selfextubation.

- A change in ICU policy can unfavorably affect selfextubation rates.

- The negative effect was found to be temporary in this study.

- Aggressive staff education may be one way to mitigate the temporary negative effect.

- Further research is needed to better elucidate all of the contributing causes of self-extubation.

\section{CONFLICT OF INTEREST}

The authors confirm that this article content has no conflict of interest.

\section{ACKNOWLEDGEMENTS}

Declared none.

\section{REFERENCES}

[1] Stawicki SP. Mechanical ventilation: weaning and extubation. OPUS 12 Scientist 2007; 1(2): 13-6.

[2] Coppolo DP, May JJ. Self-extubations: a 12-month experience. Chest 1990; 98(1): 165-9.

[3] Rothman K. Modern Epidemiology. Boston: Little Brown 1986.

[4] Schwartz DE, Matthay MA, Cohen NH. Death and other complications of emergency airway management in critically ill adults. Anesthesiology 1995; 82(2): 367-72.

[5] Vassal T, Anh NG, Gabillet JM, et al. Prospective evaluation of self-extubations in a medical intensive care unit. Intensive Care Med 1993; 19(6): 340-2.

[6] Little LA, Koeing JC Jr, Newth CJ. Factors affecting accidental extubations in neonatal and pediatric intensive care patients. Crit Care Med 1990; 18(2): 163-5.

[7] Rivera R, Tibballs J. Complicaitons of endotracheal intubation and mechanical ventilation in infants and children. Crit Care Med 1992; 20(2): 193-9.

[8] Chang LC, Liu PF, Huang YL, Yang SS, Chang WY. Risk factors associated with unplanned endotracheal self-extubation of hospitalized intubated patients: a 3-year retrospective case-control study. Appl Nurs Res 2011; 24(3): 188-92.

[9] Achauer BM, Mueller G, Vanderkam VM. Prevention of accidental extubation in burn patients. Ann Plast Surg 1997; 38(3): 280-2.

[10] Atkins PM, Mion LC, Mendelson W, Palmer RM, Slomka J, Franko T. Characteristics and outcomes of patients who selfextubate from ventilatory support: a case-control study. Chest 1997; 112(5): 1317-23.

[11] da Silva PS, Fonseca MC. Unplanned endotracheal extubations in the intensive care unit: systematic review, critical appraisal, and evidence-based recommendations. Anesth Analg 2012; 114(5): 1003-14.

[12] Christie JM, Dethlefsen M, Cane RD. Unplanned endotracheal extubation in the intensive care unit. J Clin Anesth 1996; 8(4): 28993.

[13] Curry K, Cobb S, Kutash M, Diggs C. Characteristics associated with unplanned extubations in a surgical intensive care unit. Am J Crit Care 2008; 17(1): 45-51; quiz 2. 
[14] Chevron V, Menard JF, Richard JC, et al. Unplanned extubation risk factors of development and predictive criteria for re-intubation. Crit Care Med 1998; 26(6): 1049-53.

[15] Frank BS, Lewis RJ. Experience with intubated patients does not affect the accidental extubation rate in pediatric intensive care units. Pediatr Pulmonol 1997; 23: 424-8.

[16] Mort TC. Unplanned tracheal extubation outside the operating room: a quality improvement audit of hemodynamic and tracheal airway complications associated with emergency tracheal reintubation. Anesth Analg 1998; 86(6): 1171-6.

[17] Whelan J, Simpson SQ, Levy H. Unplanned extubation. Predictors of successful termination of mechanical ventilatory support. Chest 1994; 105(6): 1808-12.

[18] Betbese AJ, Perez M, Bak E, et al. A prospective study of unplanned endotracheal extubation in intensive care unit patients. Crit Care Med 1998; 26(7): 1180-6.

[19] Huang YT. Factors leading to self-extubation of endotracheal tubes in the intensive care unit. Nurs Crit Care 2009; 14(2): 68-74.

[20] Beckmann U, Baldwin I, Durie M, et al. Problems associated with nursing staff shortage: an analysis of the first 3600 incident reports submitted to the Australian Incident Monitoring Study (AIMS ICU). Anaesth Intensive Care 1998; 26(4): 390-400.

[21] Tominaga GT, Rudzwick H, Scannell G, Waxman K. Decreasing unplanned extubations in the surgical intensive care unit. Am J Surg 1995; 170(6): 586-9.

[22] Brandstetter RD, Khawaja IT, Bartky E. Self-extubation. Chest 1991; 99(5): 1319-20.

[23] Bambi S. Accidental extubation in intensive care units: what implications for nursing care? Assist Inferm Ric 2004; 23(1): 3647.

[24] Vianna A, Rangal D, Alves A, et al. Unplanned extubation in the intensive care unit: what are the consequences? Crit Care 2007; 11(Suppl 3): P66.

[25] Jarachovic M, Mason M, Kerber K, McNett M. The role of standardized protocols in unplanned extubations in a medical intensive care unit. Am J Crit Care 2011; 20(4): 304-11.
[26] Rachman BR, Watson R, Woods N, Mink RB. Reducing unplanned extubations in a pediatric intensive care unit: a systematic approcah. Int J Pediatr 2009; 2009: 820495.

[27] Sadowski R, Dechert RE, Bandy KP, et al. Continuous quality improvement: reducing unplanned extubations in a pediatric intensive care unit. Pediatrics 2004; 114(3): 638-32.

[28] G.A. Tindol J, DiBenedetto RJ, L.Kosciuk. The role of standardized protocols in unplanned extubations in a medical intensive care unit. Am J Crit Care 1994; 20(4): 304-11.

[29] Shehabi Y, Bellomo R, Reade MC, et al. Early goal-directed sedation versus standard sedation in mechanically ventilated critically ill patients: a pilot study. Crit Care Med 2013; 41(8): 1983-91.

[30] Chia PL, Santos DR, Tan TC, Leong C, Foo D. Clinical quality improvement: eliminating unplanned extubation in the CCU. Int J Health Care Qual Assur 2013; 26(7): 642-52.

[31] Roquilly A, Cinotti R, Jaber S, et al. Implementation of an evidence-based extubation readiness bundle in 499 brain-injured patients. a before-after evaluation of a quality improvement project. Am J Respir Crit Care Med 2013; 188(8): 958-66.

[32] Xiao K, Su L, Han B, et al. Prognosis and weaning of elderly multiple organ dysfunction syndrome patients with invasive mechanical ventilation. Chinese Med J 2014; 127(1): 11-7.

[33] Zwarenstein M, Goldman J, Reeves S. Interprofessional collaboration: effects of practice-based interventions on professional practice and healthcare outcomes. Cochrane Database Syst Rev 2009; 8(3): CD000072.

[34] Chang VM. Protocol for prevention of complications of endotracheal intubation. Crit Care Nurse 1995; 15(5): 19-20, 3-7.

[35] Pesiri AJ. Two-year study of the prevention of unintentional extubation. Crit Care Nurse Q 1994; 17(3): 35-9.

[36] Carrion M, Ayuso D, Marcos M, et al. Accidental removal of endotracheal and nasogastric tubes and intravascular catheters. Crit Care Med 2000; 28: 63-6.

(C) Denny et al.; Licensee Bentham Open.

This is an open access article licensed under the terms of the Creative Commons Attribution Non-Commercial License (http://creativecommons.org/licenses/by$\mathrm{nc} / 3.0 /$ ) which permits unrestricted, non-commercial use, distribution and reproduction in any medium, provided the work is properly cited. 